\title{
The theory on thing's limits. Part 3: The root cause of modern physics' century-long wandering
}

\author{
Jian DING *
}

(Retired, Integrated Electronic Systems Lab Co. Ltd., Jinan 250100, China)

\begin{abstract}
Electromagnetic radiation can cause an electron to be further broken down, because the radiated photons have taken away a part of the static mass that originally belonged to the electron. This article makes use of the experimental data approved by HLS800MeV electron storage ring, and reasonably calculates that the static mass of each electron in the ring is roughly in the range of $1.16 \times 10^{-33}$ to $3.23 \times 10^{-35}(\mathrm{~kg})$. Once a consensus can be reached on this, it will mean the end of the century-long wandering of modern physics. If tracing its root, the problem has lain in relying solely on experimental data, like a blind person crossing the river by feeling for stones, difficult to grasp the correct research direction. So it is pointed out that based on the continuities of the development among truths and objective things are more reliable than relying solely on experimental data. Because the experimental data are not eternal, but any truth must be eternal. So once the two are combined with each other, as if the blind person has regained his vision again. That is, only based on the concepts and methods introduced in this article "The theory on thing's limits", the correct research direction can be ensured.
\end{abstract}

Key words: Synchrotron radiation; energy shrinkage; continuity; electron; photon; special relativity PACS: 41.60.Ap; 29.20.D-; 03.75.-b; 03.30.+p

\section{Introduction}

Electromagnetic radiation can cause an electron to be further broken down. The reason ${ }^{[1]}$ is that the radiated photons have taken away a part of the static mass that originally belonged to the electron. It's like a bread that becomes two parts of the same texture after a bite. Such an obvious and reasonable thing, but most physicists have gone so far as to turn a blind eye to it, let alone to reach a

* Corresponding author introduction: Jian DING, Male, Retired, metaphysics and physics. E-mail: jiandus@163.com 
consensus. To this day, they still insist that an electron cannot be further broken down. So that modern physics has been fettered here, and wandered for a century-long, with absurd theories growing, and in a dilemma.

This article is the third part of full text of "The theory on thing's limits". When quoting from the contents of the previous two parts of the full text, use "P1" or "P2" to index. As for the formula (1) in $\mathrm{P} 2$, it can be represented by "P2-1". The rest may be inferred by analogy.

In the P1 ${ }^{[2]}$, the characteristic of truth was explained, that was, it could not be proved by empirical methods, and only be approached gradually by repeated practice. And according to the principle of inertia, the norm for identifying truth was established. Based on this, we could break through the bondage of finite thinking, and extend the philosophy of materialism to the category of metaphysics. Thereby restored the true nature of metaphysics. This means that metaphysics has neither divorced from practices, nor just observed objective things with a one-sided, isolated and static way of thought. In people's daily life, everyone has used it, just not deliberately reflected on.

In the P2 ${ }^{[3]}$, based on Newton's first law, which was a basis point that could look at the overall situation, and pointed out: Only in reality could there be inertia. And mass was the measure of magnitude of an object's inertia. Therefore, where there was inertia, there must be matter and mass, and vice versa.

Inertia, which also represents the continuity of the development of things. And infer from this, as long as in reality, between any two things can certainly find a direct or indirect causality through continuity. This is an objective law that has universal significance.

\section{Modern physics in a dilemma}

According to this, we can distinguish and find out those absurd theories that have resulted in modern physics being fettered here, and wandered for a century-long, and in a dilemma.

First, as far as the uncertainty principle is concerned, it is wrong to default that an electron cannot be further broken down. Because in this principle, the development of things loses the continuity. Only in this way to judge, can we reasonably explain a large number of experimental data related to it under the premise of maintaining the continuity of the development of things.

If this truth that any experimental data were not eternal could be understood at that time, so the energy shrinkage effect that existed from high-speed electrons would be bound to be taken into 
account, and that was exactly the primary factor causing the spectrum redshift. In other words, the Big Bang theory derived based on the Doppler Effect as experimental basis has been a paradox because its research direction was wrong from the beginning.

Furthermore, three key errors in Einstein's special relativity have also been found out. One was to confuse the value $c$ of light speed in vacuum with the speed of light in reality. The second was to offend a truth that was the absoluteness of simultaneity. The third was cyclical argumentation. That was, first used the absolute space-time established by Newton as a criterion and came to the conclusion that the relative space-time was curved; then once more used the relative space-time as a criterion to change the unit length and time established by the absolute space-time.

As a truth, Newton's first law is in an ideal realm beyond the real space. Although it has been widely applied to the physical space in reality, but it belongs to the category of metaphysics, that is, the reason it exists is only in order to the existence of the natural science. This means that based on the continuities of the development among truths and objective things are more reliable than relying solely on experimental data. Because the experimental data are not eternal, but any truth must be eternal.

For example, the elementary charge and rest mass of an electron have been still defined as physical constants until now. And the bases for defining them were only the statistical values obtained from a large number of the experimental data when the electrons were moving in a state of low-speed just after they had left the atom. Since these two physical constants were statistical values, so it has been proved that the static mass of every electron is different.

Of course, there is only likeness but no absolutely identical in reality, which is a truth. Therefore, it is an inescapable objective reality that there is a small error between the static mass of every electron and its physical constant. But if this error turns out to be hundreds of times larger than the static mass of the electron itself, so its state has been from quantitative change to qualitative change. Can't help but ask, are these two physical constants still reliable?

Any so-called authoritative theory must be restricted by truth ${ }^{[4]}$ and tested by practice. The following is to make use of the experimental data submitted by the other party to demonstrate whether an electron would be further broken down, which is to result in the fundamental reason for the wandering of modern physics for a century-long. 


\section{HLS800MeV electron storage ring}

Hefei Light Source (HLS) is affiliated with the National Synchrotron Radiation Laboratory (NSRL) and located at the University of Science and Technology of China (USTC) in Hefei City, Anhui Province. Its main body is an electron storage ring ${ }^{[5]}$ that the nominal energy is $800 \mathrm{MeV}$, and the circumference is 66.1308 meters. Its structure and layout are shown in Figure 1.

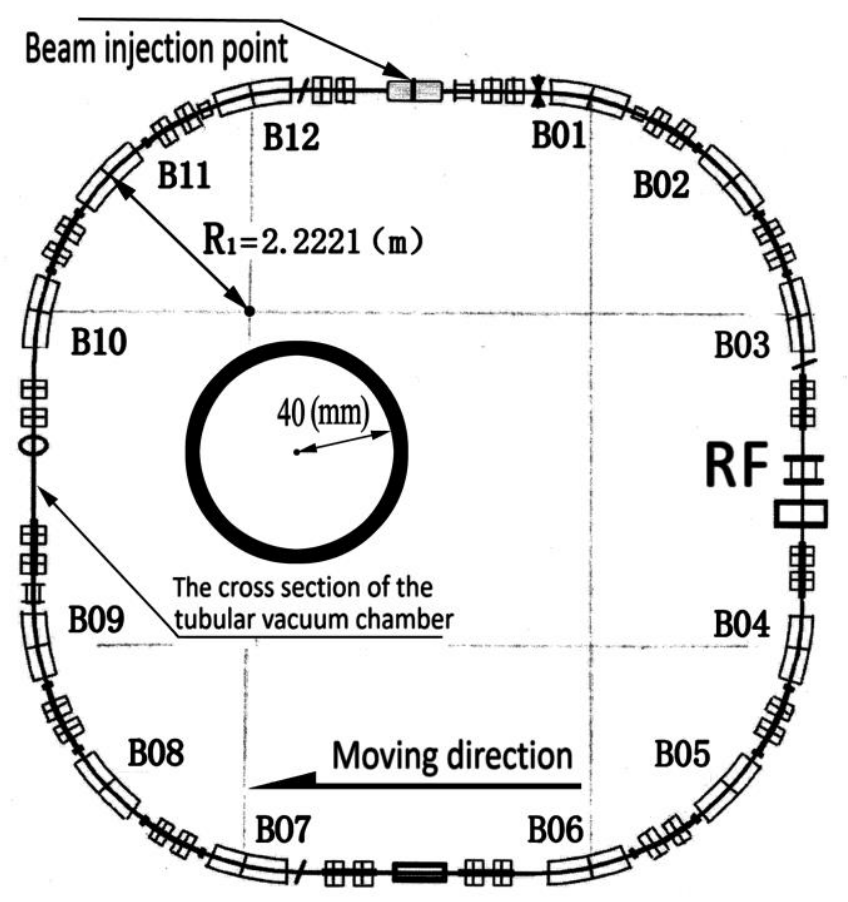

\section{Figure1. Layout diagram of HLS80OMeV electronic storage ring}

The purpose of this article is to make use of the experimental data approved by the storage ring to demonstrate that electromagnetic radiation would be bound to cause an electron to be further broken down. According to this, in Figure 1, only the beam injection point, the 12 piece of deflecting magnets $\left(\mathrm{B}_{01}-\mathrm{B}_{12}\right)$ at four corners, and the high-frequency cavity $\mathrm{RF}$, as well as the cross section of the tubular vacuum chamber are schematically marked.

As for the contents listed in Table 1 are part of its parameters, and also following this principle. Among them, all the rest can be considered as approved values that have been tested in practice except for the nominal electron energy and the number of electrons in the ring. Combined with Figure 1 and Table 1, it can be seen that in the tubular vacuum chamber, the electron beam does cyclotron motion at a speed $v$ close to the value $c$ of light speed in vacuum, and the frequency is 
4.5333MHz. These high-speed electrons are deflected 30 degrees at each piece of deflecting magnets. So their trajectory through the 12 piece of deflecting magnets is exactly a circle with a radius of $\mathrm{R}_{1}=2.2221$ meters.

Table 1. Parts of the parameters on HLS $800 \mathrm{MeV}$ electron storage ring

\begin{tabular}{ll|l}
\hline Nominal electron energy & $\mathrm{E} / \mathrm{MeV}$ & 800 \\
\hline Circulating beam intensity & $\mathrm{I} / \mathrm{mA}$ & $300 \sim 100$ \\
\hline Number of electrons in the ring & $4.14 \sim 1.38 \times 10^{11}$ \\
\hline Characteristic wavelength radiated & $\lambda_{\mathrm{p}} / \mathrm{nm}$ & 2.427 \\
\hline Curvature radius at deflecting magnets & $\mathrm{R}_{1} / \mathrm{m}$ & 2.2221 \\
\hline Magnetic induction intensity at deflecting magnets $\quad \mathbf{B}_{1} / \mathrm{T}$ & 1.2 \\
\hline Cyclotron frequency of electrons & $f_{1} / \mathrm{MHz}$ & 4.5333 \\
\hline Inner radius of the tubular vacuum chamber $\quad / \mathrm{mm}$ & 40 \\
\hline
\end{tabular}

Furthermore, when these high-speed electrons make circular motion in each piece of deflecting magnets, due to centripetal force, electromagnetic radiation will be randomly generated. In view of their moving speeds are close to the value $c$ of light speed in vacuum, also known as synchrotron radiation. As shown in figure 2.

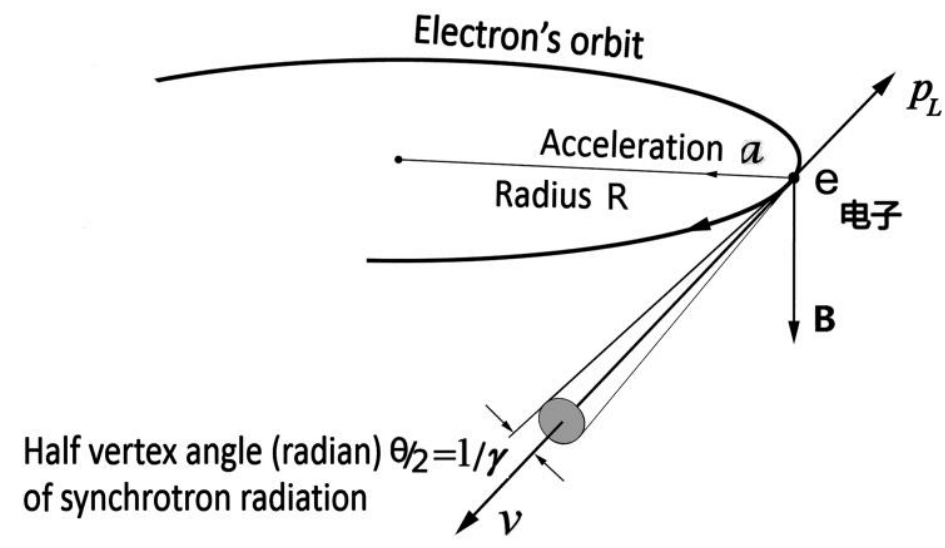

\section{Figure 2. Radiation angular distribution of moving electrons}

Synchrotron radiation ${ }^{[6]}$ is a new type of light source with high brightness, continuous spectrum, good directivity and polarization. Its spectrum can range from X-ray, vacuum ultraviolet, ultraviolet light, visible light, has been covered to infrared. As shown in Figure 2, the beam generated by synchrotron radiation is along the tangential direction of the moving electron, and concentrated in a 
very narrow cone. Its half vertex angle $\theta / 2 \approx 1 / \gamma$ (in radians) is very small, almost close to a parallel beam. For example, according to the later calculation, the expansion factor $\gamma_{1}=1564.4$ of the HLS800MeV electron storage ring, so the half vertex angle $\theta / 2 \approx 0.64$ (mrad) of the electron synchrotron radiation in the ring can be known.

\section{Radiated photons are bound to take matter away}

The fact is that every one of the photons generated by synchrotron radiation transfers a momentum $p_{L}$ (see Figure 2) in the form of an impulse to the electron emitting it, which equal to the momentum of the photon itself, but the opposite direction, thereby causes the electron's moving speed to decrease slightly. So the electrons do every turn in the storage ring, they can replenish energy when passing through the high-frequency cavity RF (see Figure 1) in order to restore to the speed before the synchrotron radiation occurred. It must be prompted here that although the speed can be restored, but the momentum and energy not be restored, because the radiated photons have taken away a part of the static mass that originally belonged to the electron.

As far as the formulas P2-1, P2-2, P2-3, P2-4 or P2-6 are concerned, the reason why momentum or energy is defined, that is a measure in order to be used for the motions of matter and the interaction between them. Objects in reality, including various particles, all can be collectively known as matter. When there is matter, there must be inertia, and the measure of magnitude of inertia is mass. As far as a photon is concerned, it has both momentum and energy, so how can it have no mass? If there is no mass, who exactly is moving? And who is the object for receiving force?

As for fluctuation, it is precisely because a photon has mass and inertia, so it can present out a continuous state of fluctuating when subjected to force. Otherwise, if the photon has no mass, there can be no inertia. According to Newton's first law, its moving trajectory will not present out continuity. And such a photon does not exist in reality. That is to say, any photon must have mass.

Therefore, every photon generated by synchrotron radiation would be bound to take away a part of the static mass that originally belonged to the electron. That is to say, the energy shrinkage effect presented by high-speed electrons, its root lies in that an electron can be further broken down. In this regard, there has been no consensus. And this is exactly the objective fact that modern physics must face. Any so-called authoritative statement that has attempted to circumvent it vaguely, all will 
certainly come back here again, be tested and reconfirmed. Otherwise, modern physics will be yet fettered here and difficult to make great progress.

For more than a hundred years, physicists have generally believed that an electron would not be further broken down. Of course, the same has been true for the deciders, developers and users of the storage ring. For example, when measured that the beam intensity of circulating electrons dropped from $300(\mathrm{~mA})$ to $100(\mathrm{~mA})$, they believed that the number of electrons in the ring had dropped from $4.14 \times 10^{11}$ to $1.38 \times 10^{11}$.

In other words, in Table 1, the default values of the charge $e \equiv e_{0}$ and static mass $m_{0} \equiv M_{0}$ of an electron were all using physical constants. Among them, $e_{0}=-1.602 \times 10^{-19}(C)$ was the elementary charge of the electron, and $M_{0}=9.11 \times 10^{-31}(\mathrm{~kg})$ was the rest mass of the electron, including the charge-to-mass ratio $e_{0} / M_{0}$ of the two, which were all physical constants.

And it is these physicists, most of them would still agree that photons should have mass when asking themselves. It can be seen that their hearts have been ambivalent about whether an electron will be further broken down. In view of this, we should seriously reflect, carefully verify, and exorcise the devils in own heart in order to facilitate the consensus. This is crucial for the future direction of physics.

\section{The key lies in the invariance of the charge-to-mass ratio}

According to the kinetics formulas (P2-3, P2-4 and P2-5) of Einstein's special relativity, it can be known that wherever there is energy, there must be mass, and vice versa. Therefore, due to the impact of electromagnetic radiation, the energy $\Delta E=\Delta m_{0} c^{2} \gamma$ that every radiated photon has would be bound to take away a part of the static mass $\Delta m_{0}$ that originally belonged to the electron. It shows that there is the energy shrinkage effect for high-speed electrons. Therefore, the charge $e$ and static mass $m_{0}$ of every electron have been no longer constants. But their charge-to-mass ratio

$$
e / m_{0}=e_{0} / M_{0}=1.7588 \times 10^{11}(C / k g)
$$

has always remained the same physical constant value.

This shows that electrons and the photons radiated by electrons all are composed of the same substance. Just like a piece of bread becomes two parts after being got a bite. Although they are 
different in size, but have the same texture. It is obvious things, and makes sense. So it is emphasized again that the charge-to-mass ratio $e / m_{0}$ of an electron refers to the ratio of its charge to the quantity of matter, which is not affected by relativistic effects and electromagnetic radiation. By the same token, this conclusion applies not only to all electrons, also applies to all photons with the same charge-to-mass ratio as electrons, including those photons they will radiate again.

In view of this, the formula P2-7 is relisted as follows:

$$
\beta=\frac{v}{c}=\sqrt{\frac{\left(\frac{e}{m_{0}}\right)^{2}}{\left(\frac{e}{m_{0}}\right)^{2}+\left(\frac{c}{\boldsymbol{B} R}\right)^{2}}}
$$

It can be seen that when the magnetic induction intensity $\mathbf{B}$ and the curvature radius $\mathrm{R}$ have been designed to the given values, if the impact of electromagnetic radiation is not considered, the moving speed $v$ of every electron in the storage ring is also a given value, and has nothing to do with its mass. According to this, once it is proved that synchrotron radiation would be bound to cause every electron in the ring to be further broken down, it means that their charge-to-mass ratio $e / m_{0}$ always remains the same physical constant value.

Substituting the curvature radius $\mathrm{R}=\mathrm{R}_{1}=2.2221(\mathrm{~m})$ and the magnetic induction intensity $\mathbf{B}=\mathbf{B}_{1}=1.2(\mathrm{~T})$ of the electrons moving at the deflecting magnets in Table 1 into the formula (1), the moving speed

$$
v_{1}=\beta_{1} c=0.999999795695 c
$$

of electrons in the storage ring can be calculated. Among them, $c=299792458(\mathrm{~m} / \mathrm{s})$ is the value of light speed in vacuum. From this, the expansion factor

$$
\gamma_{1}=1 / \sqrt{1-v_{1}^{2} / c^{2}}=1564.4
$$

can be calculated. Then substitute it into formula P2-3, and the static mass of the electron is according to the designer's default value $m_{0}=M_{0}$, and calculate out the energy of every electron in the storage ring as $E=m_{0} c^{2} \gamma_{1}=1.28 \times 10^{-10}(J)$, which can be converted to $800(\mathrm{MeV})$. In other words, the Hefei Light Source was defined as the HLS800MeV electron storage ring, which has clearly expressed that its designers defaulted that an electron would not be further broken down.

The particles in reality cannot be in a state that Newton's first law describes as unaffected by 
forces. Therefore, random electromagnetic radiation will be generated, which is an inevitable physical phenomenon. Due to the impact of electromagnetic radiation, every one of high-speed electrons in the storage ring can be further broken down, thereby the energy shrinkage effect will be presented. This means that not only the so-called $800(\mathrm{MeV})$ is just the nominal value of the energy of every electron in the ring, but also the static mass of every electron injected into the ring from the linear accelerator is far less than their values of physical constants. So, what exactly is the static mass of every electron in the ring?

\section{The critical state of the beam lifetime}

In August 2014, the chief engineer Li Weimin, who was in charge of the accelerator transformation project at the time, there was a speech draft ${ }^{[7]}$ when introducing the debugging situation of Hefei Light Source (HLS). Among them, two pictures (Figure 3 and Figure 4) has been extracted from the data related to the beam lifetime.

Figure 3 was in the debugging process for the track repeatability, two relatively complete decay process curves of the beam intensity were intercepted from the real-time monitoring chart during July 25 to 28 . Among them, the two ellipses marked by a and b were added in order to draw attention of the two decay curves in the areas.

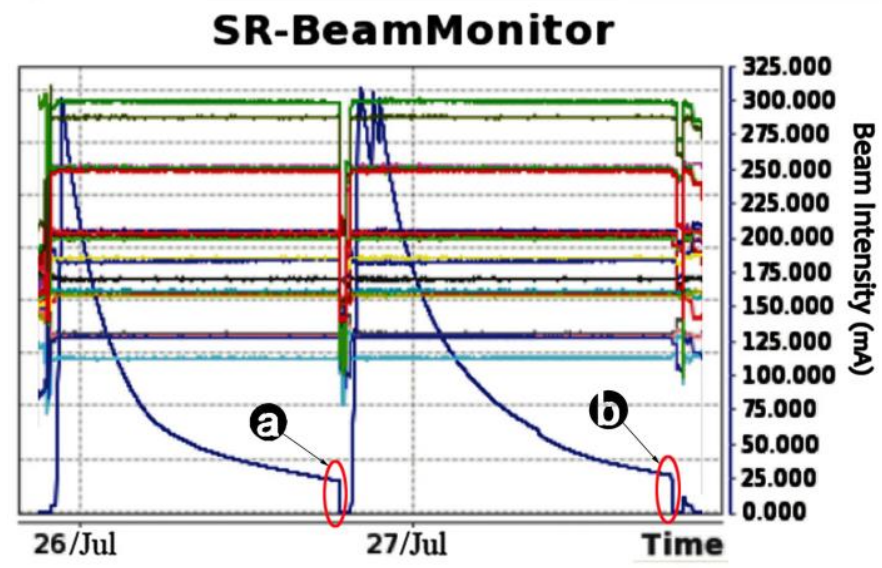

Figure 3. Beam lifetime (A)

(From the lecture of introducing the debugging situation of HLS80OMeV)

The process described by these two curves showed that the static mass of each electron in the ring had decayed below a specific critical value. This meant that every photon generated by synchrotron radiation after it transferred own momentum in the opposite direction, $p_{L}=h / \lambda_{p}$, to the corresponding electron, would very likely cause the electron to hit the inner wall of the tubular 
vacuum chamber. So all the electrons in the ring would be lost in a very short time.

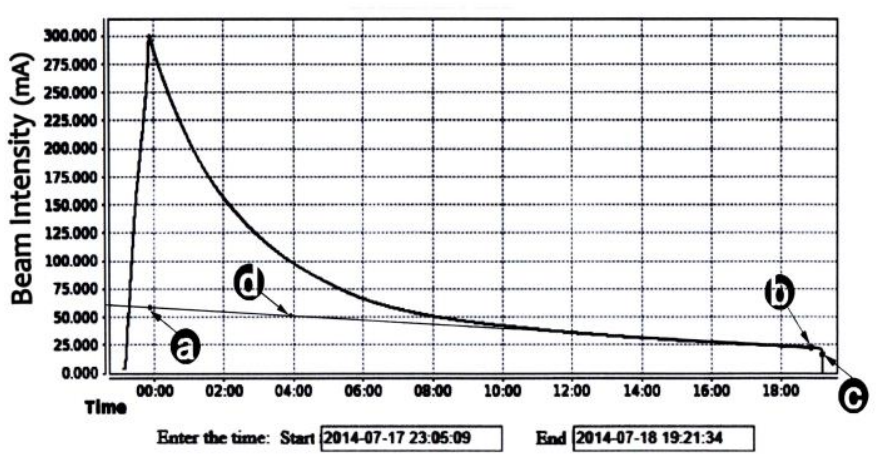

Figure 4. Beam lifetime (B)

(From the lecture of introducing the debugging situation of $\mathrm{HLS} S 800 \mathrm{MeV}$ )

Figure 4 was also in the debugging process for the track repeatability, a complete decay process curve of the beam intensity was traced based on the real-time monitoring chart during July 17 to 18 . Among them, a, b, c, d and the four points marked by them, as well as the auxiliary straight line ab, all are added for facilitate the subsequent explanation.

It can be seen from Figure 4 that in the HLS800MeV storage ring, the time for the beam intensity to drop from $300(\mathrm{~mA})$ to $20(\mathrm{~mA})$ was about 19 hours. After that, the beam intensity quickly dropped to $0(\mathrm{~mA})$, in less than half an hour.

According to this, it can be considered that all the electrons that had not been maintained to point $b$, had hit the inner wall of the tubular vacuum chamber to be lost due to various reasons at different moments during the 19 hours. Considering that synchrotron radiation had the characteristics of microscopic disorder and macroscopic order, as long as the electrons that could be maintained to the point $b$, the slope of their static masses to decay was just like the straight line from the point a to point $b$, should be approximately linear. This meant that the auxiliary straight line ab represented the change values of the beam intensity of all electrons that could be maintained to the point in the ring.

So it can be considered that from the point $b$ to point $c$, the decay of every electron's static mass has exceeded the critical value. In other words, synchrotron radiation caused the moving speed of these electrons to decrease more and more, so that they could not smoothly pass through the 12 piece of deflecting magnets and return to the high-frequency cavity RF to replenish energy. So, in less than half an hour, more and more electrons would hit the inner wall of the tubular vacuum chamber until they were all lost. 


\section{Calculate the static mass of electron in the ring}

In Figure 1 and Table 1, the schematic diagram of the tubular vacuum chamber and the dimensions of the cross section were given. It can be seen that with the decrease of moving speed of electrons, when the curvature radius of passing through the deflecting magnets dropped to

$$
\mathrm{R}_{2}=2.2221-0.04=2.1821(\mathrm{~m}),
$$

they would hit the inner wall of the tubular vacuum chamber.

Substituting $\mathrm{R}=\mathrm{R}_{2}=2.1821(\mathrm{~m})$ and $\mathrm{B}=\mathrm{B}_{1}=1.2(\mathrm{~T})$ into formula (1), the moving speed

$$
v_{2}=\beta_{2} c=0.999999788136 c
$$

of an electron that just hit the inner wall of the tubular vacuum chamber could be calculated. And its expansion factor

$$
\gamma_{2}=1 / \sqrt{1-v_{2}^{2} / c^{2}}=1536.4
$$

In the storage ring, when high-speed electrons pass through the deflecting magnets, synchrotron radiations were generated. The spectra of the radiated photons were continuous. It can be seen from Table 1 that the photons with characteristic wavelengths $\lambda_{p}=2.427(\mathrm{~nm})$ were included among them. And at the point $\mathrm{b}$, the static mass $m_{0 b}$ of every electron had been decayed and reached nearby the critical value.

Then, as far as a typical electron among them is concerned, in the process of passing through the 12 pieces of deflecting magnets, since 12 photons with characteristic wavelengths $\lambda_{p}=2.427(\mathrm{~nm})$ were radiated, so its moving speed had been dropped to near $v_{2}$, and even hit the inner wall of the tubular vacuum chamber to be lost. According to the principle of conservation of momentum, the following equation

$$
m_{0 b} v_{1} \gamma_{1}-\left(m_{0 b}-12 \Delta m_{0 b}\right) v_{2} \gamma_{2}=12 h / \lambda_{p}
$$

should hold. Among them, $p_{b 1}=m_{0 b} v_{1} \gamma_{1}$ was the momentum of an electron at the point $\mathrm{b}$, and $p_{b 2}=\left(m_{0 b}-12 \Delta m_{0 b}\right) v_{2} \gamma_{2}$ was the momentum that after the electron radiated 12 photons of characteristic wavelengths $\lambda_{\mathrm{p}}=2.427(\mathrm{~nm})$ to cause its moving speed to drop near $v_{2}$. And $p_{L}=h / \lambda_{p}$ was the momentum of each radiated photon, where $h$ Planck's constant. 
Since the static mass of an electron was much larger than that of a photon $\left(m_{0 b}>\Delta m_{0 b} \times 10^{6}\right)$, so the term $\Delta m_{0 b}$ could be ignored. Thus, the above equation is simplified to

$$
m_{0 b}\left(v_{1} \gamma_{1}-v_{2} \gamma_{2}\right)=12 h / \lambda_{\mathrm{p}}
$$

Substituting $\lambda_{\mathrm{p}}=2.427(\mathrm{~nm})$ and the relevant values calculated above, the static mass

$$
m_{0 b}=\frac{12 h / \lambda_{p}}{v_{1} \gamma_{1}-v_{2} \gamma_{2}} \approx 3.88 \times 10^{-34}(\mathrm{~kg})
$$

of an electron near the point $\mathrm{b}$ could be obtained. Again according to de Broglie matter wave relation P2-1, the wavelength

$$
\lambda_{b}=\frac{h}{m_{0 b} v_{1} \gamma_{1}}=3.64 \times 10^{-12}(m)
$$

of the electrons near the point $\mathrm{b}$ could also be calculated.

So, the unknown can be obtained through the known based on the calculating formula

$$
I_{\mathrm{x}}=N_{x} f_{x} m_{0 x}\left(e / m_{0}\right)
$$

of the beam intensity in the ring. Among them, $N_{x}$ is the total number of electrons, $f_{x}$ is the cyclotron frequency of electrons, $m_{0 x}$ is the static mass of the electron. And $e / m_{0}$ is the charge-to-mass ratio of electrons, which is a physical constant.

Given $f_{1}=f_{x}$ in Table 1 , the beam intensity $I_{b}=I_{x}(0.02 \mathrm{~A})$ at the point $\mathrm{b}$ in Figure 4 , and the $m_{0 b}=m_{0 x}$ that calculated above. Then, it could be calculated that the total number of electrons at the point $\mathrm{b}$ was $6.46 \times 10^{13}$ (See Table 2$)$.

Table 2. The change values of the electrons in the ring following the beam intensity

\begin{tabular}{|c|c|c|c|}
\hline Beam intensity ratio & Static mass $/ \mathrm{kg}$ & Actual electron number & wavelength $/ \mathrm{m}$ \\
\hline $300 / 60$ & $1.16 \times 10^{-33}$ & $3.23 \times 10^{14}$ & $1.21 \times 10^{-12}$ \\
\hline $100 / 50$ & $9.70 \times 10^{-34}$ & $1.29 \times 10^{14}$ & $1.46 \times 10^{-12}$ \\
\hline $20 / 20$ & $3.88 \times 10^{-34}$ & $6.46 \times 10^{13}$ & $3.64 \times 10^{-12}$ \\
\hline
\end{tabular}

\section{Compared with the approved values in Table 1}

In Table 2, when the beam intensity decayed to 20 (mA), the static mass of every electron also 
decayed to $3.88 \times 10^{-34}(\mathrm{~kg})$, and the total number was $6.46 \times 10^{13}$. This corresponded to the data at the point $\mathrm{b}$ in Figure 4, which has been calculated previously. Combined with Figure 4, it can be seen that the $6.46 \times 10^{13}$ electrons, when they were at the point a, the beam intensity generated was about 60 (mA). According to formula (2), it can be calculated that the static mass of every electron should be $1.16 \times 10^{-33}(\mathrm{~kg})$. This means that the static mass of every electron injected from the linear accelerator into the storage ring should be close to this value. That is, the static mass has been greatly decayed to near $1 / 783$ of the nominal value.

At this moment, the beam intensity in the ring was 300 (mA). According to formula (2), it is calculated that there were a total of $3.23 \times 10^{14}$ such electrons in the ring. Which could be converted into $4.13 \times 10^{11}$ nominal electrons, and corresponded to the number of electrons when the beam intensity was $300(\mathrm{~mA})$ in Table 1.

When these electrons had looped in the ring for 19 hours and reached the point $b$, there were left with only $6.46 \times 10^{13}$ electrons, whose beam intensity decayed along the straight line ab. And the rest of the electrons, all had hit the inner wall of the tubular vacuum chamber respectively to be lost due to various reasons at different moments during the 19 hours.

Therefore, the so-called "beam intensity ratio" in Table 2 referred to the ratio of the beam current intensity generated by all the electrons in the ring, at a certain moment in Figure 4, to that generated by these $6.46 \times 10^{13}$ electrons.

For another example, in Table 2, when the beam intensity in the ring was $100(\mathrm{~mA})$, the beam intensity generated by the $6.46 \times 10^{13}$ electrons was $50(\mathrm{~mA})$, which corresponded to the point $\mathrm{d}$ in Figure 4. According to formula (2), it can be calculated that when the beam intensity ratio was $100 / 50$, the same was these $6.46 \times 10^{13}$ electrons, due to the impact of electromagnetic radiation, the static mass of every one of them had decayed along the slope of straight line ab to $9.70 \times 10^{-34}(\mathrm{~kg})$, the beam intensity generated was $50(\mathrm{~mA})$. And at this moment, the beam intensity in the ring was $100(\mathrm{~mA})$. Again according to formula (2), it is calculated that the total number of electrons in the ring was also reduced to $1.29 \times 10^{14}$. Which could be converted into $1.38 \times 10^{11}$ nominal electrons, and corresponded to the number of electrons when the beam intensity was 100 (mA) in Table 1.

\section{A few notes}

In Figure 4, the electron near the point $c$ can be defined like this way that when it passing 
through a certain deflecting magnet, as long as to radiate a photon of the characteristic wavelength $\lambda_{\mathrm{c}}=2.427(\mathrm{~nm})$, its moving speed could be dropped to near $v_{2}$. Such an electron was almost impossible to return again to the high-frequency cavity RF to replenish energy. Therefore, it would hit the inner wall of the tubular vacuum chamber to be lost in a very short time.

According to the above derivation, its static mass

$$
m_{\mathrm{Oc}}=m_{\mathrm{ob}} / 12 \approx 3.23 \times 10^{-35}(\mathrm{~kg})
$$

and wavelength

$$
\lambda_{c}=\frac{h}{m_{0 c} v_{1} \gamma_{1}}=4.37 \times 10^{-11}(m)
$$

can also be easily calculated. This means that during the period from the point $\mathrm{b}$ to point $\mathrm{c}$, the static mass of every electron was roughly in this interval from $m_{0 b}$ to $m_{0 c}$. And once entering this interval, in less than half an hour, all the electrons would hit the inner wall of the tubular vacuum chamber, and showed as the beam intensity to drop to 0 (mA).

In Table 2, when the beam intensity in the ring was $300(\mathrm{~mA})$, the wavelength of electrons was $1.21 \times 10^{-12}(\mathrm{~m})$, which was also calculated according to formula P2-1. So it can be seen that the static mass of every electron in the storage ring was roughly in the range of $1.16 \times 10^{-33}$ to $3.23 \times 10^{-35}(\mathrm{~kg})$ during the entire decaying process of the beam intensity. As for its corresponding wavelength, which was roughly in this range of $1.21 \times 10^{-12}$ to $4.37 \times 10^{-11}(\mathrm{~m})$.

If you still insist that an electron cannot be further broken down, just use the beam of electrons injected into the storage ring by the linear accelerator to do interference or diffraction experiments, and no need to quantify accurately, only need by roughly determining the wavelength, the right or wrong will be distinguished lightly. Because if an electron really cannot be further broken down, its wavelength according to formula P2-1 should be near $1.55 \times 10^{-15}(\mathrm{~m})$, which compares with every one of electrons injected into the storage ring by the linear accelerator, the difference between the two wavelengths is about 783 times. And if the electron beam at the point $b$ is used for interference or diffraction experiments, the difference will be expanded to about 2348 times.

Finally, it is pointed out that if the discussion in this article is accepted, after verification and reaching a consensus, we will be bound to face the current situation that is absurd phenomena growing and hard to get rid of ingrained habits. But more importantly, we should draw lessons from 
the wandering for a century-long. That is to say, an effective method should be found as soon as possible in order to correctly grasp the research direction. And the concepts and methods introduced in this article "The theory on thing's limits", as well as the norm for identifying truth, especially the discussion on the continuity of the development of things, have constituted an effective solution.

\section{Conclusion}

Electromagnetic radiation can cause an electron to be further broken down, because the radiated photons have taken away a part of the static mass that originally belonged to the electron. This article makes use of the experimental data approved by HLS800MeV electron storage ring, and reasonably calculates that the static mass of each electron in the ring is roughly in the range of $1.16 \times 10^{-33}$ to $3.23 \times 10^{-35}(\mathrm{~kg})$, and the corresponding wavelength of $1.21 \times 10^{-12}$ to $4.37 \times 10^{-11}(\mathrm{~m})$.

Such an obvious and reasonable thing, but most physicists go so far as to turn a blind eye to it, let alone reach a consensus. To this day, they still insist that an electron cannot be further broken down. So that modern physics has been fettered here, and wandered for a century-long, with absurd theories growing, and in a dilemma.

If tracing its root, the problem lies in relying solely on experimental data, which is like a blind person crossing the river by feeling for stones, difficult to grasp the correct research direction. So it is pointed out that based on the continuities of the development among truths and objective things are more reliable than relying solely on experimental data. Because the experimental data are not eternal, but any truth must be eternal.

Therefore, as long as the two are combined with each other, it is as if the blind person has regained his vision. And the concepts and methods introduced in this article "The theory on thing's limits", as well as the norm for identifying truth, especially the discussion on the development of things with continuity, have provided an effective solution for how to grasp the correct research direction.

\section{References}

[1] Ding J. A brief analysis of the research scheme of the cyclotron radiation from a single electron. Phys Astron Int J. 2020;4(2): 60-64. DOI: 10.15406/paij.2020.04.00202

[2] DING, Jian. 2021. "The Theory on Thing's Limits. Part 1: The Norm of Identifying Truth.” OSF Preprints. May 11. doi:10.31219/osf.io/gh4vk. 
[3] DING, Jian. 2021. "The Theory on Thing's Limits. Part 2: A Brief Analysis of the New Knowledge of Newton's First Law.” OSF Preprints. June 29. doi:10.31219/osf.io/ytxfs.

[4] Jian DING. The Research of Using Truth to Restrict Authoritative Theories. Journal of Philosophy and Ethics. 2020; 2(1): 43-50.

[5] He D. Hefei National Synchrotron Radiation Source [J]. Physics, 1992, 21(5): 257-262. (in Chinese)

[6] Jin Y M. Electron Storage Ring Physics [M]. Hefei: USTC Press, 2001: 5-7. (in Chinese)

[7] Li W M. Summarizing the beam emittance of storage ring. August, 2004. (in Chinese)

http://www.doc88.com/p-77439951081168.html. 


\title{
物极理论 3：近代物理学百年非徊的根源
}

\author{
丁健* \\ 积成电子股份有限公司（已退休） 中国济南 250100
}

\begin{abstract}
捕要: 电磁辐射可以导致电子被进一步地分解, 因为辐射出的光子带走了原本属于电子的一部分静质量。 本文利用HLS800MeV电子储存环已核准的实验数据, 合理地计算出环中每个电子的静质量大约在 $1.16 \times 10^{-30}$ 至3. $23 \times 10^{-35}(\mathrm{~kg})$ 这个范围内。一旦能够就此形成共识, 将意味着近代物理学百年徘徊的结束。若究其根 源, 问题在于仅依赖实验数据, 就好像是盲人在摸着石头过河, 难以把握正确的研究方向。于是指出, 立 足于真理与客观事物之间发展的连续性, 要比仅依赖实验数据更为可靠。因为实验数据不是永恒的, 但任 何真理都必定是永恒的。所以一旦两者结合在一起, 就如同盲人又恢复了视力。也就是说, 只有依据本文 《物极理论》中所介绍的理念和方法, 才能确保正确的研究方向。
\end{abstract}

关链词: 电磁辐射; 能量收缩; 连续性; 电子; 光子; 狭义相对论

中国分类号: $0434.11 ; 0572.21+1 ; 0413.1 ; 0412.1$

* 作者简介：丁健（1953-）, 男, 已退休。主要研究方向: Metaphysics and physics... E-mail: jiandus@163.com 